\title{
On a significant slowing-down of the kinetics of fast transient processes in a fast reactor ${ }^{*}$
}

\author{
Gennady G. Kulikov ${ }^{1}$, Anatoly N. Shmelev², Vladimir A. Apse², Evgeny G. Kulikov² \\ 1 State Atomic Energy Corporation ROSATOM, 24 Bolshaya Ordynka Str., 119017 Moscow, Russia \\ 2 National Research Nuclear University MEPhI, 31 Kashirskoe Hwy, 115409 Moscow, Russia \\ Corresponding author: Evgeny G. Kulikov (egkulikov@mephi.ru)
}

Academic editor: Yury Kazansky • Received 24 December 2019 Accepted 12 August 2020 • Published 20 November 2020

Citation: Kulikov GG, Shmelev AN, Apse VA, Kulikov EG (2020) On a significant slowing-down of the kinetics of fast transient processes in a fast reactor. Nuclear Energy and Technology 6(4): 295-298. https://doi.org/10.3897/nucet.6.60379

\begin{abstract}
The kinetics of nuclear reactors is determined by the average neutron lifetime. When the inserted reactivity is more than the effective delayed neutron fraction, the reactor kinetics becomes very rapid. It is possible to slow down the fast reactor kinetics by increasing the neutron lifetime. The authors consider the possibility of using the lead isotope, ${ }^{208} \mathrm{~Pb}$, as a neutron reflector with specific properties in a lead-cooled fast reactor. To analyze the emerging effects in a reactor of this type, a point kinetics model was selected, which takes into account neutrons returning from the ${ }^{208} \mathrm{~Pb}$ reflector to the reactor core.

Such specific properties of ${ }^{208} \mathrm{~Pb}$ as the high atomic weight and weak neutron absorption allow neutrons from the reactor core to penetrate deeply into the ${ }^{208} \mathrm{~Pb}$ reflector, slow down in it, and have a noticeable probability to return to the reactor core and affect the chain fission reaction. The neutrons coming back from the ${ }^{208} \mathrm{~Pb}$ reflector have a long 'deadtime', i.e., the sum of times when neutrons leave the reactor core, entering the ${ }^{208} \mathrm{~Pb}$ reflector, and then diffuse back into the reactor core. During the 'dead-time', these neutrons cannot affect the chain fission reaction. In terms of the delay time, the neutrons returning from the deep layers of the ${ }^{208} \mathrm{~Pb}$ reflector are close to the delayed neutrons. Moreover, the number of the neutrons coming back from the ${ }^{208} \mathrm{~Pb}$ reflector considerably exceeds the number of the delayed neutrons.

As a result, the neutron lifetime formed by the prompt neutron lifetime and the 'dead-time' of the neutrons from the ${ }^{208} \mathrm{~Pb}$ reflector can be substantially increased. This will lead to a longer reactor acceleration period, which will mitigate the effects of prompt supercriticality. Thus, the use of ${ }^{208} \mathrm{~Pb}$ as a neutron reflector can significantly improve the fast reactor nuclear safety.
\end{abstract}

\section{Keywords}

Kinetics, fast reactor, reflectors based on natural lead and lead-208, VVER and CANDU thermal reactors, fission chain reaction, transient process, asymptotic acceleration

\section{Introduction}

It is shown in (Shmelev et al. 2011, 2013, Kulikov et al. 2014, 2017a, b, c, d, Kulikov 2017) that the use in a fast reactor of a physically thick reflector about $4 \mathrm{~m}$ thick made of lead-208 instead of a reflector made of natural lead $0.5 \mathrm{~m}$ thick makes it possible to radically slow down the development of a fission chain reaction. This is due

* Russian text published: Izvestiya vuzov. Yadernaya Energetika (ISSN 0204-3327), 2020, n. 3, pp. 41-49.

Copyright Kulikov GG et al. This is an open access article distributed under the terms of the Creative Commons Attribution License (CC-BY 4.0), which permits unrestricted use, distribution, and reproduction in any medium, provided the original author and source are credited. 


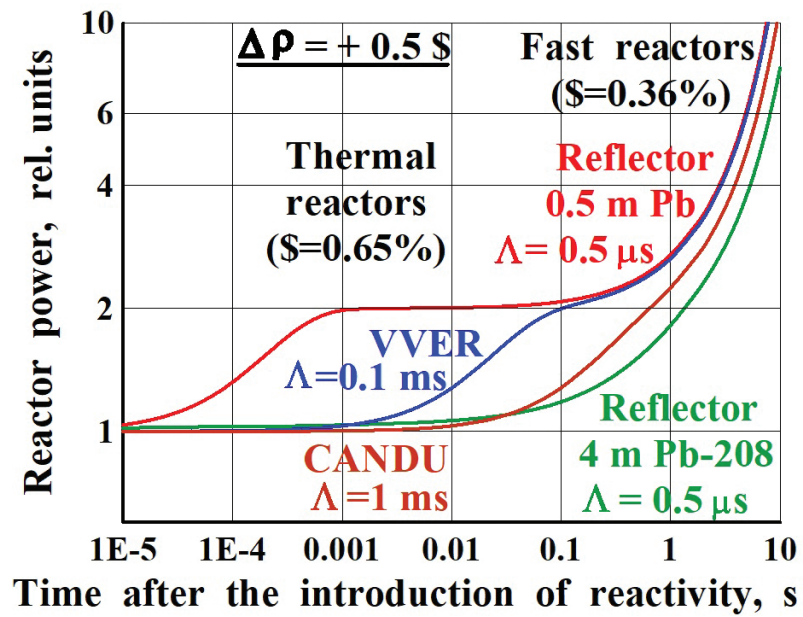

Figure 1. Change in the power of various reactors depending on the time after the introduction of reactivity $+0.5 \$$.

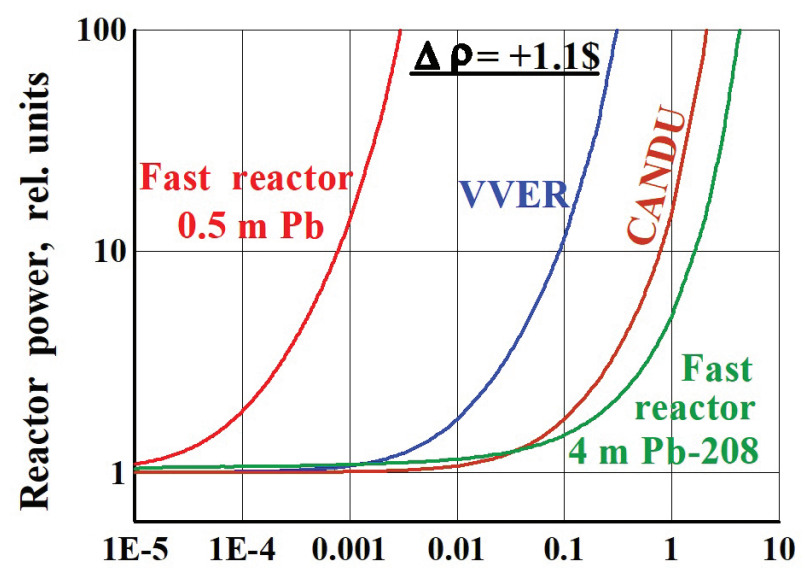

Time after the introduction of reactivity, $s$

Figure 2. Change in the power of various reactors depending on the time after the introduction of reactivity $+1.1 \$$.

to the long return of neutrons to the core from a reflector made of lead-208 due to its heavy atomic weight and low neutron absorption. As a result, the average neutron lifetime in such a reactor is about three orders of magnitude longer than in a reactor with a natural lead reflector. In the event of an unauthorized insertion of positive reactivity, the reactor power growth slows down and the amount of released energy in a reactivity accident decreases, which should improve its nuclear safety. The physical features of the formation and development of a fission chain reaction in a reactor of this type are considered in (Kulikov et al. 2018a, b, c, d)

An analysis of fast reactors with both reflector types was carried out. Their main characteristics are given in Table 1. The effective delayed neutron fraction is taken equal to $0.36 \%$, i.e., the same as for plutonium fuel.

For comparison, the most widespread thermal reactors VVER and CANDU - are considered under the same conditions. For them, the effective delayed neutron fraction is taken equal to $0.36 \%$, i.e., the same as for uranium fuel. The average neutron lifetime in these reactors differs by
Table 1. Characteristics of reactors and their asymptotic acceleration periods $\left(T_{\mathrm{ac}}\right)$ at different introduced reactivity.

\begin{tabular}{|c|c|c|c|c|c|}
\hline \multirow[t]{3}{*}{ Reactor } & \multirow{3}{*}{$\begin{array}{c}\text { Reflector } \\
\text { (thickness, } \\
\text { material) }\end{array}$} & \multirow{3}{*}{$\begin{array}{c}\text { Effective } \\
\text { delayed } \\
\text { neutron } \\
\text { fraction } \$, \%\end{array}$} & \multirow{3}{*}{$\begin{array}{c}\text { Average } \\
\text { neutron } \\
\text { lifetime, ms }\end{array}$} & \multicolumn{2}{|c|}{ Reactivity, \$ } \\
\hline & & & & +0.5 & +1.1 \\
\hline & & & & \multicolumn{2}{|c|}{$\mathbf{T}_{\mathrm{ac}}, \mathrm{s}$} \\
\hline BREST & $0.5 \mathrm{~m}, \mathrm{~Pb}$ & 0.36 & 0.0005 & 5.72 & 0.00128 \\
\hline VVER & - & 0.65 & 0.1 & 5.80 & 0.11 \\
\hline CANDU & - & 0.65 & 1.0 & 6.50 & 0.61 \\
\hline BREST & $4 \mathrm{~m}, \mathrm{~Pb}-208$ & 0.36 & +0.5 & 7.61 & 1.15 \\
\hline
\end{tabular}

about an order of magnitude, i.e., 0.1 and $1 \mathrm{~ms}$, respectively. Note that in a domestic thermal reactor of the RBMK type, the lifetime is comparable to that of CANDU.

The kinetics in the transient process and during the asymptotic acceleration of thermal reactors was calculated within the point model (Bell and Glasstone 1970, Bekurtz and Wirtz 1968) taking into account six groups of delayed neutrons, and for a fast reactor - using multipoint kinetics. In this case, one point fell on the core with delayed neutrons, and the other points - on each reflector layer $0.5 \mathrm{~m}$ thick (Shmelev et al. 2011). The kinetic parameters (the fraction and time of neutron return from the reflector through its layers) were estimated using TIME26, a one-dimensional 26-group program for calculating fast reactors (Apse and Shmelev 2008), within the framework of spherical geometry.

\section{Features of power growth and energy release in the transient reactor acceleration process}

Figs 1, 2 show the change in the power of a fast reactor surrounded by a $0.5 \mathrm{~m}$ thick reflector made of natural lead and a $4 \mathrm{~m}$ thick reflector made of lead-208, as well as thermal reactors of the VVER and CANDU types, depending on the time after the insertion of positive reactivities of 0.5 and $1.1 \$$, respectively.

It can be seen that at a reactivity of $\$ 0.5$ in a fast reactor with a reflector made of natural lead, an almost instantaneous (in about $1 \mathrm{~ms}$ ) double power leap occurs. It is associated with an improvement in the conditions for neutron multiplication and occurs due to the multiplication of prompt neutrons, the lifetime of which is short (about $0.4 \mu \mathrm{s}$ ). However, there is practically no further power growth until a time instant of about $0.1 \mathrm{~s}$. This is due to the fact that the reactor remains subcritical on prompt neutrons. Therefore, its acceleration requires also delayed neutrons, the contribution of which to the fission chain reaction begins to appear after about $0.1 \mathrm{~s}$, since this corresponds to the lifetime of the shortest-lived group of delayed neutrons. A further power growth occurs with the simultaneous participation of both prompt and delayed neutrons with an asymptotic period of $5.72 \mathrm{~s}$ in developing the chain fission reaction (see Table 1). All this corresponds to the well-known model of kinetics without feedback (Bell and Glasstone 1970, Bekurtz and Wirtz 1968). 
In a VVER-type reactor, a similar change in power occurs with the only difference that the average prompt neutron lifetime is about two orders of magnitude longer than in a fast reactor. Therefore, a double power leap is observed after the reactivity is inserted (after about $0.1 \mathrm{~s}$ ). Immediately thereafter, delayed neutrons begin to contribute to the fission chain reaction. In this regard, the section of the plateau in power depending on the time after introduction of reactivity is weakly expressed.

In a CANDU-type reactor, the leap in prompt neutron power occurs even later (after about $0.8 \mathrm{~s}$ ), and the plateau section is absent, since the average lifetime of prompt neutrons is still an order of magnitude closer to the lifetime of delayed neutrons.

In a fast reactor with a lead-208 reflector, the power leap is completely absent, since the lifetime of neutrons returning from the reflector to the core corresponds to a wide range of values from the lifetime of prompt neutrons in the core (for neutrons returning from the first layers of the reflector) to the lifetime of delayed neutrons (for neutrons that return from the depth of the reflector), and the fraction of neutrons in the reflector is large (about \$ 35). Table 1 shows that, although the asymptotic acceleration period gradually increases from reactor to reactor, this increase is insignificant.

The picture changes dramatically when a positive reactivity of $+1.1 \$$ is inserted, which exceeds the effective delayed neutron fraction (Fig. 2). The reactors become supercritical on prompt neutrons, the average lifetime of which is significantly shorter than that of delayed neutrons; therefore, the power grows much faster with clearly shorter asymptotic acceleration periods (see Table 1), which radically differ in magnitude for different reactors. Thus, the asymptotic acceleration period for a fast reactor with a natural lead reflector is approximately $1 \mathrm{~ms}$ and, with a lead-208 reflector, it is almost three orders of magnitude longer. For thermal reactors, the asymptotic acceleration periods are intermediate values and, in a CANDU-type reactor, it is several times longer than in a VVER-type reactor, since the average prompt neutron lifetime in CANDU is about an order of magnitude longer than in VVER (see Table 1).

However, as is known, in reactivity accidents, damage to core elements can be caused not only by a power leap but also by the amount of energy released in this element. Figs 3, 4 show the amount of excess energy, i.e. the energy that does not have time to be removed under the condition of normal heat removal in different reactors from the time after the introduction of positive reactivity of 0.5 and $1.1 \$$, respectively. It is assumed that excess energy is released when the reactor power exceeds its rated value. Excess energy is measured in relative units as the product of the rated power and time. Thus, for example, excess power equal to 10 relative units means that energy was released in the fuel, and it was not removed to the coolant; this energy is generated in a reactor operating at a tenfold increase in the rated power for $1 \mathrm{~s}$, or a fivefold increase in the rated power for $2 \mathrm{~s}$, etc.

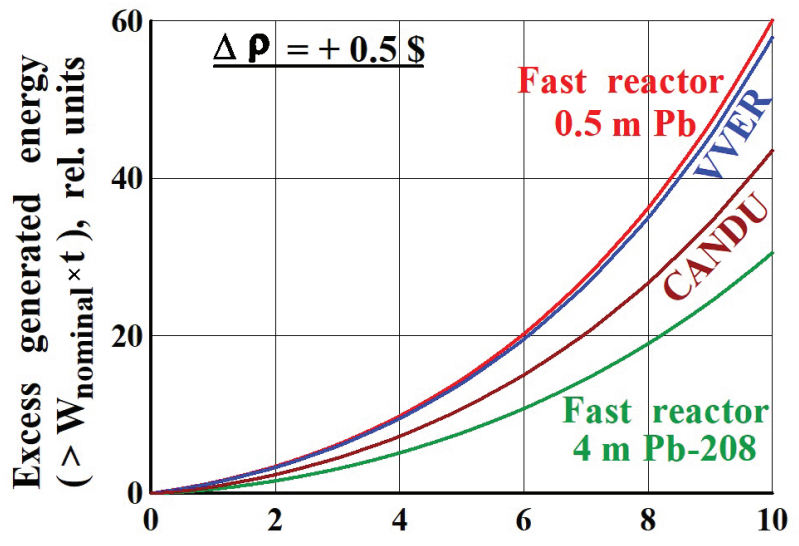

Time after the introduction of reactivity, $s$

Figure 3. The amount of excess (not removed from the fuel) energy in various reactors versus time after the introduction of reactivity $+0.5 \$$.

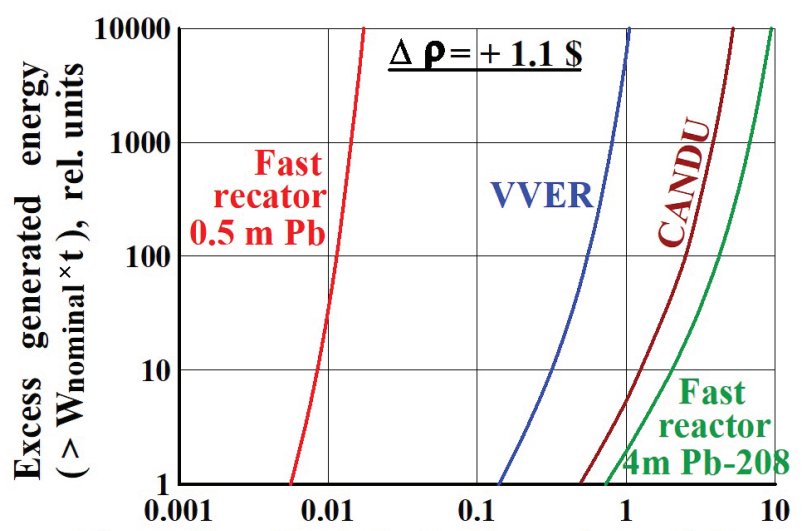

Time after the introduction of reactivity, $s$

Figure 4. The amount of excess (not removed from the fuel) energy in various reactors versus time after the introduction of reactivity $+1.1 \$$.

Fig. 3 shows that, at inserted positive reactivity of $0.5 \$$, the amount of energy not removed from the fuel in a fast reactor with a lead-208 reflector is approximately two times less than with a natural lead reflector at any time after the insertion of reactivity. In this sense, a VVER-type reactor is close to a fast reactor with a natural lead reflector, and CANDU occupies an intermediate position between fast reactors with different reflectors. It is obviously due to the corresponding asymptotic reactor acceleration periods (see Table 1). Thus, the rate of generation of excess energy does not differ too much in different reactors, since the acceleration process in them occurs mainly on delayed neutrons, the lifetime of which weakly depends on fuel types.

However, the rate of generation of excess energy differs sharply in reactors when positive reactivity is inserted that exceeds the effective delayed neutron fraction. As Fig. 4 shows, the same value (for example, 100 relative units) of excess energy in different reactors (fast reactor with a natural lead reflector, VVER, CANDU, fast reactor with a lead-208 reflector) is reached for significantly 
different times $(0.0114,0.55,2.5$ and $4.2 \mathrm{~s}$, respectively). This is due to significantly different asymptotic reactor acceleration periods (see Table 1). The asymptotic periods are determined by the absence of long-lived neutrons in a natural lead reflector in a fast reactor, the presence of comparatively longer-lived prompt neutrons in a VVER, the presence of even longer-lived prompt neutrons in CANDU, and, finally, the presence of the longest-lived neutrons in a lead-208 reflector in a fast reactor.

\section{Conclusion}

It is shown that due to the long return of neutrons from a physically thick reflector made of lead-208 to the core of a fast reactor, its kinetics is drastically slowed down. This leads to a significant power growth slowing-down during the insertion of positive reactivity in comparison with the same reactor, but with a reflector made of natural lead, and even in comparison with thermal reactors of the VVER and CANDU type, which are traditionally charac-

\section{References}

- Apse VA, Shmelev AN (2008) Use of the Computer Code TIME26 in the Training Design of Fast Reactors and Accelerator Driven Systems. Moscow, NRNU MEPhI Publ., 63 pp. [in Russian]

- Bekurtz K, Wirtz K (1968) Neutron Physics. Moscow, Atomizdat Publ., 456 pp. [in Russian]

- Bell GJ, Glasstone S (1970) Nuclear Reactor Theory. Van Nostrand Reinhold Company, 494 pp.

- Kulikov EG (2017) Radiogenic Lead as Coolant, Reflector and Moderator in Advanced Fast Reactors. Journal of Physics: Conference Series 781(1): e012002. https://doi.org/10.1088/1742 6596/781/1/012002

- Kulikov GG, Apse VA, Kulikov EG, Kozhahmet BK, Shkodin AO, Shmelev AN (2017a) Radiogenic lead from poly-metallic thorium ores as a valuable material for advanced nuclear facilities. Kerntechnik 82(1): 87-91. https://doi.org/10.3139/124.110665

- Kulikov GG, Apse VA, Shmelev AN, Kulikov EG (2017b) Improved Safety Fast Reactor with "Reservoir" for Delayed Neutrons Generating. Journal of Physics: Conference Series 781(1): e012009. https:// doi.org/10.1088/1742-6596/781/1/012009

- Kulikov GG, Shmelev AN, Apse VA, Kulikov EG (2017c) On fundamental Quality of Fission Chain Reaction to Oppose Rapid Runaways of Nuclear Reactors. Journal of Physics: Conference Series 781(1): e012006. https://doi.org/10.1088/1742-6596/781/1/012006

- Kulikov GG, Shmelev AN, Apse VA, Kulikov EG (2018a) Models of the In-Hour Equation for Fast Reactors Surrounded by a Physically Thick Neutron Reflector for Upgrading the Reactor Safety. Journal of Physics: Conference Series 1133(1): e012036. https://doi. org/10.1088/1742-6596/1133/1/012036

- Kulikov GG, Shmelev AN, Apse VA, Kulikov EG (2018b) Safety of a fast reactor with a reflector containing a moderator with a high atomic weight and low neutron absorption. In: Proceedings of the $5^{\text {th }}$ International Scientific and Technical Conference on Innovative terized by slower kinetics as compared to fast reactors. As a result, the generation of energy not removed from the fuel to the coolant is significantly slowed down. All this means that such a reactor is safer in terms of nuclear accidents.

Richard Feynman called fast assembly a 'slumbering dragon', meaning the catastrophically rapid development of a fission chain reaction even when the value of inserted reactivity approaches the effective delayed neutron fraction. The use of a physically thick lead-208 reflector makes it possible to divert this 'sword of Damocles' from fast reactors not only when the reactivity approaches but even exceeds the value of the effective delayed neutron fraction.

\section{Acknowledgements}

This work was supported by the Ministry of Science and Higher Education of the Russian Federation as part of the Project No. 13.9748.2017/8.9.
Projects and Technologies of Nuclear power. Moscow, NIKIET Publ. [in Russian]

- Kulikov GG, Shmelev AN, Apse VA, Ternovykh MYu, Kulikov EG (2014) Favorable features in kinetics of fast reactors with physically thick reflector containing ${ }^{208} \mathrm{~Pb}$. In: Proceedings of the International Conference PHYSOR-2014. The Role of Reactor Physics Toward a Sustainable Future. Kyoto, Japan.

- Kulikov GG, Shmelev AN, Kulikov EG, Apse VA (2018c) Nuclear safety of the fast reactor with ${ }^{208} \mathrm{~Pb}$-reflector at neutron flash above effective fraction of delayed neutrons. Journal of Physics: Conference Series 1133(1): e012037. https://doi.org/10.1088/17426596/1133/1/012037

- Kulikov GG, Shmelev AN, Kulikov EG, Apse VA (2018d) Peculiarities of the Fast Reactor with Reflector from Lead-208. Journal of Physics: Conference Series 1133(1): e012038. https://doi. org/10.1088/1742-6596/1133/1/012038

- Kulikov GG, Shmelev AN, Kulikov EG, Apse VA, Chubko NV (2017d) The Role of Neutrons from the Reflector of a Fast Reactor in Increasing the Resistance of the Fission Chain Reaction to Rapid Runaway. Atomnaya Energiya [Atomic Energy] 123(6): 351-352. https://doi.org/10.1007/s10512-018-0363-z [in Russian]

- Shmelev AN, Kulikov GG, Apse VA, Kulikov EG, Artisyuk VV (2011) Radiogenic Lead with Dominant Content of 208Pb: New Coolant and Neutron Moderator for Innovative Nuclear Facilities. Internet Journal of Hindawi Publishing Corporation: Science and Technology of Nuclear Installations 2011: e252903. https://doi. org/10.1155/2011/252903

- Shmelev AN, Kulikov GG, Kryuchkov EF, Apse VA, Kulikov EG (2013) Application of Radiogenic Lead with Dominant Content of ${ }^{208} \mathrm{~Pb}$ for Long Prompt Neutron Lifetime in Fast Reactor. Nuclear Technology 183(3): 409-426. https://doi.org/10.13182/ NT13-A19429 